\title{
INTERSECTION PROPERTIES OF POLYHEDRAL NORMS
}

\author{
J. P. MORENO AND R. SCHNEIDER
}

\begin{abstract}
We investigate the family $\mathcal{M}$ of intersections of balls in a finite dimensional vector space with a polyhedral norm. The spaces for which $\mathcal{M}$ is closed under Minkowski addition are completely determined. We characterize also the polyhedral norms for which $\mathcal{M}$ is closed under adding a ball. A subset $\mathcal{P}$ of $\mathcal{M}$ consists of the Mazur sets $K$, defined by the property that for any hyperplane $H$ not meeting $K$ there is a ball containing $K$ and not meeting $H$. We characterize the Mazur sets in terms of their normal cones and also as summands of closed balls. As a consequence, we characterize the polyhedral spaces with only trivial Mazur sets as those whose unit ball is indecomposable.
\end{abstract}

\section{INTRODUCTION}

The present paper is a sequel to [15] and is part of a program initiated in recent years to investigate the basic algebraic/topological properties of the family $\mathcal{M}$ of all intersections of closed balls in a normed space $X$. This family has appeared in problems from convex geometry, geometry of Banach spaces, fixed point theory, and combinatorial geometry. An early result concerns the Mazur intersection property, dating back to Mazur [12]. The space $X$ has this property if $\mathcal{M}$ is equal to the family of all closed bounded convex subsets of $X$. It was proved by Phelps [17] that a finite dimensional space has the Mazur intersection property if and only if the set of extreme points of the dual unit ball $B^{*}$ is dense in the boundary of $B^{*}$. An infinite dimensional generalization is due to Giles, Gregory and Sims [7]. Our aim is to establish the basic facts concerning $\mathcal{M}$ when the space lacks the Mazur intersection property. In this context, it is known that $\mathcal{M}$ is uniformly very porous [10] and that it need not be (topologically) closed [15].

Two kinds of problems are considered in this paper, those connected with Minkowski sums and those pertaining to Mazur sets. Recall that $\mathcal{M}$ is (i) stable if $\overline{C+D} \in \mathcal{M}$ whenever $C, D \in \mathcal{M}$; (ii) ball stable if $\overline{C+B} \in \mathcal{M}$ for every $C \in \mathcal{M}$, where $B$ is the unit ball of the space. These properties introduced in [8] have close connections with the continuity of the ball hull mapping [15]. The closed bounded convex set $K$ is said to be a Mazur set if the following separation property is satisfied: for every hyperplane $H$ with positive distance from $K$, there exists a ball $D$ with $K \subset D$ and $H \cap D=\emptyset$. The family

2000 Mathematics Subject Classification: 52A21, 46B20

The first autor was partially supported by the DGICYT project BFM 2003-06420. The second author is partially supported by the European Network PHD, FP6 Marie Curie Actions, RTN, Contract MCRN511953.

Key words and phrases. Intersections of balls, ball stability, polyhedral norm, Mazur set, Mazur space. 
of all Mazur sets in our normed space is denoted by $\mathcal{P}$. Observe that $\mathcal{P}$ is contained in $\mathcal{M}$. The space is called a Mazur space if $\mathcal{P}=\mathcal{M}$. Basic results concerning Mazur sets and Mazur spaces can be found in [8], [9]. For instance, $\mathcal{M}$ is stable in every Mazur space. In this paper, we restrict ourselves to finite dimensional polyhedral spaces, that is, finite dimensional real vector spaces with a norm whose unit ball is a polytope. In this elementary situation, several questions about the classes $\mathcal{M}$ and $\mathcal{P}$ have explicit answers.

It is known that $\mathcal{M}$ is stable in $\ell_{\infty}$ and in every two-dimensional space, thus it should not come as a surprise that it is also stable in

$$
X=X_{1} \oplus_{\infty} \cdots \oplus_{\infty} X_{k}
$$

where each $X_{i}$ is a normed space of dimension at most two. More surprising is the fact that the converse is true when the norm is polyhedral: every finite dimensional polyhedral space with stable $\mathcal{M}$ is obtained in this way. Finite dimensional polyhedral spaces which are direct sums of the form (1) have recently come up in connection with the investigation of suns in normed spaces [4].

Next we characterize the finite dimensional polyhedral normed spaces for which $\mathcal{M}$ is ball stable. The characterization involves relations between the normal cones of the unit ball $B$ of the space. It should be observed that the normal cones of the unit ball $B$ are in correspondence with the exposed faces of the dual unit ball $B^{*}$, thus this characterization can alternatively be formulated in terms of the facial structure of $B^{*}$, as in the characterization result of Phelps.

In the last section, we are concerned with spaces in which the only Mazur sets are balls and one-pointed sets. Typical examples are the spaces $\ell_{1}^{d}$, for $d>2$ [9]. We improve this result by obtaining a complete characterization of the finite dimensional polyhedral spaces satisfying this property. The notion of indecomposability plays a major role in this result. As a main step to achieve this characterization, the Mazur sets in finite dimensional polyhedral spaces are completely determined. Our characterization is in terms of normal fans, on the one hand, and summands of the unit ball, on the other. Using this result, we are able to characterize the finite dimensional polyhedral Mazur spaces $X$ as those for which $\mathcal{M}$ is stable.

\section{Preliminaries}

Throughout this paper, $X$ is a real normed space of dimension $1<d<\infty$, with norm $\|\cdot\|$. Its unit ball, dual space, and dual unit ball are denoted by $B,\left(X^{*},\|\cdot\|^{*}\right)$, and $B^{*}$, respectively. A homothet of $B$, that is, any set of the form $\lambda B+t$ with $\lambda>0$ and $t \in X$, is called a ball. The family of all nonempty intersections of balls is denoted by $\mathcal{M}_{X}$, or briefly by $\mathcal{M}$, when no ambiguity arises. By $\mathcal{K}$ we denote the set of all nonempty compact convex subsets of $X$. 
The support function of $K \in \mathcal{K}$ is defined by $h(K, f):=\max _{x \in K} f(x)$ for $f \in X^{*}$. The functional $f \in X^{*} \backslash\{0\}$ supports $K$ at $x$ if $f(x)=h(K, f)$, that is, if $f$ attains its maximum on $K$ at the point $x$. We write

$$
H^{-}(K, f):=f^{-1}(-\infty, \max f(K)]=\{x \in K: f(x) \leq h(K, f)\}
$$

for the (closed) supporting halfspace of $K$ determined by $f \in X^{*} \backslash\{0\}$, and

$$
H(K, f):=\{x \in K: f(x)=h(K, f)\}
$$

for the supporting hyperplane of $K$ determined by $f$. The set $F(K, f):=K \cap H(K, f)$ is the (exposed) face of $K$ determined by $f$. The dual unit sphere, that is, the boundary of $B^{*}$, is denoted by $S^{*}$. In connection with intersections of balls in Banach spaces, a certain subset of the dual unit sphere plays an important role. This is the set of semi-denting points, which in the finite-dimensional case coincide with the limits of exposed points of $B^{*}$ (see [15], Remark after Corollary 3, and observe that every extreme point is a limit of exposed points, by the theorem of Straszewicz ([18], Theorem 1.4.7)). We denote by $\Omega$ the closure of the set of exposed points of $B^{*}$. The following description of the intersections of balls (see [15], (7)) will be useful.

Lemma 2.1. Let $K \in \mathcal{K}$. Then $K \in \mathcal{M}$ if and only if $K=\bigcap_{f \in \Omega} H^{-}(K, f)$.

If $X$ has a polyhedral norm, then $\Omega$ is just the set of vertices of the dual unit ball $B^{*}$, or, equivalently, the set of all norm one functionals $f \in X^{*}$ for which $f^{-1}(1)$ is a facet (a face of codimension one) of the polytopal unit ball $B$. If $K \in \mathcal{M}$ is a polytope and $f \in S^{*}$ is such that $F(K, f)$ is a facet of $K$, then it follows from Lemma 2.1 that $f \in \Omega$.

In the following, we make essential use of support cones and normal cones, which describe the behavior of a closed convex set at one of its points. We recall briefly the definitions. Let $K \subset X$ be a closed convex set and $x \in K$. The support cone $S(K, x)$ of $K$ at $x$ is the closure of the set $\{\lambda(y-x): y \in K, \lambda \geq 0\}$. It is a closed convex cone and can also be represented as

$$
S(K, x)=\bigcap_{x \in H(K, f)} H^{-}(K, f)-x,
$$

the intersection extending over all $f \in S^{*}$ for which $x \in H(K, f)$ (if $x$ is an interior point of $K$, the intersection of the empty family of subsets of $X$ is understood as $X$ ). The dual convex cone

$$
N(K, x)=S(K, x)^{*}=\left\{f \in X^{*}: f(z) \leq 0 \forall z \in S(K, x)\right\}
$$

is called the normal cone of $K$ at $x$. Evidently,

$$
N(K, x)=\left\{f \in X^{*}: f(y) \leq f(x) \forall y \in K\right\},
$$


thus $f \in N(K, x)$ if and only if $f$ supports $K$ at $x$ or $f$ is the zero functional.

A closed convex cone $C$ is called a normal cone of $K$ if there exists a point $x$ in the boundary of $K$ such that $C=N(K, x)$. If $K$ is a polytope, $F$ is a face of $K$ and $x$ is a relatively interior point of $F$, then the normal cone $N(K, x)$ does not depend on the choice of $x$. It is denoted by $N(K, F)$ and called the normal cone of $K$ at $F$, thus $N(K, F)=\left\{f \in X^{*}: F \subset F(K, f)\right\}$. Its dimension satisfies $\operatorname{dim} F+\operatorname{dim} N(K, F)=d$. The finite system of all normal cones of a polytope is called the normal fan of the polytope.

If $x$ is a boundary point of the unit ball $B$, then

$$
N(B, x) \cap S^{*}=\left\{f \in S^{*}: f(x)=1\right\},
$$

which is an exposed face of the dual unit ball $B^{*}$. Thus, the normal cones of the unit ball are precisely the positive hulls of the exposed faces of the dual unit ball. The following result will be needed.

Lemma 2.2. Let $K, L \subset X$ be nonempty closed convex sets.

(a) If $x \in K$ and $y \in L$, then

$$
N(K+L, x+y)=N(K, x) \cap N(L, y) .
$$

(b) If $K, L$ are polytopes and $x \in K \cap L$, then

$$
N(K \cap L, x)=N(K, x)+N(L, x) .
$$

For a proof, we refer to [18], Theorem 2.2.1 (the use of a scalar product in [18] is evidently not essential for the validity of these results). However, (b) is formulated there for general convex bodies, which requires an extra condition. That this condition can be deleted in the case of polytopes, follows from the fact that for a polytope $K$ the set $\{\lambda(y-x): y \in K, \lambda \geq 0\}$ is already closed and hence equal to the support cone $S(K, x)$. This, in turn, implies that for polytopes $K, L$ and $x \in K \cap L$ we have $S(K \cap L, x)=S(K, x) \cap S(L, x)$, from which (b) follows.

\section{StABility AND BALL STABILITY OF POLYHEDRAL NORMS}

The purpose of this section is to obtain characterizations for both the stability and the ball stability of $\mathcal{M}$ in finite dimensional polyhedral spaces. We begin with a result concerning $\ell_{\infty}$ sums.

Proposition 3.1. Let $X_{1}, \ldots, X_{k}$ be finite dimensional normed spaces, let

$$
X=X_{1} \oplus_{\infty} \cdots \oplus_{\infty} X_{k} .
$$

Then $\mathcal{M}_{X}$ is stable (ball stable) if and only if each $\mathcal{M}_{X_{i}}$ is stable (ball stable). 
Proof. We will write the proof only for stability, since for ball stability the argument is similar. Let $B_{i}$ be the unit ball of $X_{i}(i=1, \ldots, k)$, then $B=B_{1} \oplus \cdots \oplus B_{k}$ is the unit ball of $X$. Let $\pi_{i}: X \rightarrow X_{i}$ denote the canonical projection. We claim that

$$
K \in \mathcal{M}_{X} \Leftrightarrow K=K_{1} \oplus \cdots \oplus K_{k} \text { with } K_{i} \in \mathcal{M}_{X_{i}}, \quad i=1, \ldots, k .
$$

Indeed, if $K \in \mathcal{M}_{X}$, say $K=\bigcap_{\alpha \in I}\left(\lambda_{\alpha} B+t_{\alpha}\right)$, then

$$
\begin{aligned}
K & =\bigcap_{\alpha \in I}\left[\left(\lambda_{\alpha} B_{1}+\pi_{1}\left(t_{\alpha}\right)\right) \oplus \cdots \oplus\left(\lambda_{\alpha} B_{k}+\pi_{k}\left(t_{\alpha}\right)\right)\right] \\
& =\left(\bigcap_{\alpha \in I}\left(\lambda_{\alpha} B_{1}+\pi_{1}\left(t_{\alpha}\right)\right)\right) \oplus \cdots \oplus\left(\bigcap_{\alpha \in I}\left(\lambda_{\alpha} B_{k}+\pi_{k}\left(t_{\alpha}\right)\right)\right) .
\end{aligned}
$$

Conversely, let $K=K_{1} \oplus \cdots \oplus K_{k}$ with $K_{i} \in \mathcal{M}_{X_{i}}, i=1, \ldots, k$. Let $x \in X \backslash K$. There is an index $j$ with $\pi_{j}(x) \notin K_{j}$. Hence, there are $\lambda>0$ and $t_{j} \in X_{j}$ with $K_{j} \subset \lambda B_{j}+t_{j}$ and $\pi_{j}(x) \notin \lambda B_{j}+t_{j}$. Here $\lambda$ can be chosen arbitrarily large (if $t_{j}$ is suitably adapted), in particular so large that $K_{i} \subset \lambda B_{i}$ for $i \neq j$. Then it follows that $K \subset \lambda B+t$ for $t$ with $\pi_{j}(t)=t_{j}$ and $x \notin \lambda B+t$. This proves that $K \in \mathcal{M}_{X}$, as desired.

For the proof of the proposition, suppose now that $\mathcal{M}_{X_{i}}$ is stable for $i=1, \ldots, k$. Let $C, D \in \mathcal{M}_{X}$. Using (2), we can write $C=C_{1} \oplus \cdots \oplus C_{k}$ and $D=D_{1} \oplus \cdots \oplus D_{k}$ with $C_{i}, D_{i} \in \mathcal{M}_{X_{i}}, i=1, \ldots, k$, hence

$$
C+D=\left(C_{1}+D_{1}\right) \oplus \cdots \oplus\left(C_{k}+D_{k}\right) .
$$

Since $C_{i}+D_{i} \in \mathcal{M}_{X_{i}}$, it follows from (2) that $C+D \in \mathcal{M}_{X}$. Thus, $\mathcal{M}_{X}$ is stable. Conversely, suppose that $\mathcal{M}_{X}$ is stable. Let $C_{1}, D_{1} \in \mathcal{M}_{X_{1}}$, say. There is a family $\left\{\lambda_{\alpha} B_{1}+t_{\alpha}\right\}$ of balls in $X_{1}$ with $C_{1}=\bigcap_{\alpha}\left(\lambda_{\alpha} B_{1}+t_{\alpha}\right)$. Put $\lambda:=\inf _{\alpha} \lambda_{\alpha}$. Then

$$
C_{1} \oplus \lambda B_{2} \oplus \cdots \oplus \lambda B_{k}=\bigcap_{\alpha}\left[\left(\lambda_{\alpha} B_{1}+t_{\alpha}\right) \oplus \lambda_{\alpha} B_{2} \oplus \cdots \oplus \lambda_{\alpha} B_{k}\right] \in \mathcal{M}_{X}
$$

Similarly, there is $\mu \geq 0$ with $D_{1} \oplus \mu B_{2} \oplus \cdots \oplus \mu B_{k} \in \mathcal{M}_{X}$. Since $\mathcal{M}_{X}$ is stable, we have

$$
\left(C_{1}+D_{1}\right) \oplus(\lambda+\mu) B_{2} \oplus \cdots \oplus(\lambda+\mu) B_{k} \in \mathcal{M}_{X}
$$

hence the claim (2) gives $C_{1}+D_{1} \in \mathcal{M}_{X_{1}}$. Thus $\mathcal{M}_{X_{1}}$ is stable.

Remark. The natural question whether Proposition 3.1 has an analog for $\ell_{1}$-sums has a negative answer, since $\mathcal{M}_{\ell_{1}^{3}}$ is not stable [8] (yet it is ball stable, for instance by Theorem 3.3 below). Moreover, we show that the $\ell_{1}$-sum of two finite dimensional normed spaces, both satisfying ball stability, need not be ball stable. Consider for $X$ the space $\mathbb{R}^{2}$ endowed with a norm whose unit ball is a hexagon, and let $Y=X \oplus_{1} \mathbb{R}$. That $\mathcal{M}$ is not ball stable in this space, can be deduced from Theorem 3.3. 
For finite dimensional polyhedral spaces, we are able to give an explicit description of the spaces satisfying stability.

Theorem 3.2. Let $X$ be a finite dimensional space with polyhedral norm. Then $\mathcal{M}_{X}$ is stable if and only if $X=X_{1} \oplus_{\infty} \cdots \oplus_{\infty} X_{k}$, where each $X_{i}$ is a polyhedral normed space of dimension at most two, or, equivalently, if the unit ball of $X$ is a direct sum of convex polygons of dimensions one and two.

Proof. Sufficiency is a direct consequence of Proposition 3.1 and the fact that $\mathcal{M}$ is stable in every two dimensional space [8], so it remains to prove necessity. Recall that $\operatorname{dim} X=d$ and that $\Omega$ is the set of vertices of the dual unit ball $B^{*}$. Let $\Lambda$ be the system of lines in $X^{*}$ spanned by the pairs of antipodal points in $\Omega$. A $(d-1)$-dimensional subspace of $X^{*}$ spanned by $d-1$ lines of $\Lambda$ is called a spanned subspace.

Claim. If $d-1$ spanned subspaces intersect in a line, then this line belongs to $\Lambda$.

For the proof, let $S_{1}, \ldots, S_{d-1}$ be spanned subspaces that intersect in a line $L$. Let $f_{L} \in S^{*}$ be one of the two vectors (in $X^{*}$ ) spanning $L$. We may assume that $f_{1}, \ldots, f_{d-1} \in$ $\Omega$ are $d-1$ linearly independent vectors spanning $S_{1}$. Then $f_{L} \in \operatorname{lin}\left\{f_{1}, \ldots, f_{d-1}\right\}$, and without loss of generality (replacing $f_{i}$ by $-f_{i}$ where necessary) we can assume that $f_{L} \in \operatorname{pos}\left\{f_{1}, \ldots, f_{d-1}\right\}$. The functional $f_{i}$ determines a unique facet $F_{i}=F\left(B, f_{i}\right)=$ $f_{i}^{-1}(1)$ of the polytopal unit ball $B$. Choose $x_{i} \in \operatorname{relint} F_{i}$ (the relative interior of $F_{i}$ ) $(i=1, \ldots, d-1)$. Then

$$
P_{1}:=\bigcap_{i=1}^{d-1}\left(B-x_{i}\right)
$$

is a polytope and, obviously, an element of $\mathcal{M}$. The set $G_{1}:=\bigcap_{i=1}^{d-1}\left(F_{i}-x_{i}\right)$ is a face of $P_{1}$. Since $x_{i} \in \operatorname{relint} F_{i}$, the set $G_{1}$ has the same dimension as $\bigcap_{i=1}^{d-1} f_{i}^{-1}(1)$, which is 1 , due to the linear independence of $f_{1}, \ldots, f_{d-1}$. Thus $G_{1}$ is an edge of $P_{1}$. The normal cone $N\left(P_{1}, G_{1}\right)$ of $P_{1}$ at $G_{1}$ is the positive hull of the vectors $f_{1}, \ldots, f_{d-1}$, as follows from Lemma 2.2(b), hence $f_{L} \in N\left(P_{1}, G_{1}\right)$. Performing the same construction with $S_{2}, \ldots, S_{d-1}$, we obtain polytopes $P_{1}, \ldots, P_{d-1} \in \mathcal{M}$ and corresponding edges $G_{i} \subset P_{i}$ such that $f_{L} \in \bigcap_{i=1}^{d-1} N\left(P_{i}, G_{i}\right)$. The sum $G:=G_{1}+\cdots+G_{d-1}$ is a face of $P:=P_{1}+\cdots+P_{d-1}$ and, by Lemma 2.2(a), $N(P, G)=\bigcap_{i=1}^{d-1} N\left(P_{i}, G_{i}\right)$. Observe that $N\left(G_{i}, 0\right)=S_{i}$ and, hence

$$
N(G, 0)=N\left(G_{1}+\cdots+G_{d-1}, 0\right)=\bigcap_{i=1}^{d-1} S_{i} .
$$

Since $S_{1}, \ldots, S_{d-1}$ intersect in a $\operatorname{line}, \operatorname{dim} N(G, 0)=1$. It follows that $\operatorname{dim} G=d-1$, thus $G$ is a facet of $P$. From $f_{L} \in N(P, G)$ we see that $f_{L}$ is a functional that determines $G$. Since $\mathcal{M}$ is closed under Minkowski sums, we have $P \in \mathcal{M}$. Now it follows from Lemma 2.1 that $f_{L} \in \Omega$, which proves the claim.

The proof can now be completed by applying a result from projective geometry. The following argument was essentially used before in [19], but for greater clarity we include 
it here in a simpler form. Let $P^{d-1}$ be the $(d-1)$-dimensional real projective space, whose points are the lines through 0 in $X^{*}$ and whose $k$-dimensional subspaces are the $(k+1)$-dimensional linear subspaces of $X^{*}$. Then $\Lambda$ is a system of $n$ points in $P^{d-1}$ (where $2 n$ is the number of points in $\Omega$ ), not all lying in a hyperplane. By results of Motzkin $[16,4.3]$ and Hansen [11] (showing that Motzkin's hypothesis $U_{d}$ is always satisfied), the following holds. The number of hyperplanes in $P^{d-1}$ determined by the points of $\Lambda$ is at least $n$, and it is exactly $n$ if and only if $(*)$ the points are, for odd $d-1$, on $d / 2$ lines, and for even $d-1$, all but one on $(d-1) / 2$ lines. Now suppose that $(*)$ does not hold. Then the points of $\Lambda$ determine at least $n+1$ hyperplanes in $P^{d-1}$, and these determine, by the dual of Motzkin's result, at least $n+1$ intersection points and thus at least one point not in $\Lambda$. This contradicts the claim. Thus $(*)$ holds. For the points of $\Omega$ this means that they lie, for even $d$, on $d / 2$ two-dimensional linear subspaces of $X^{*}$, and for odd $d$, on $(d-1) / 2$ two-dimensional linear subspaces and a one-dimensional linear subspace. Since $\Omega$ spans $X^{*}$, it follows that $B$ is a direct sum of two-dimensional polygons and, for odd $d$, a segment.

Remark. As noted in [15], the sets in $\mathcal{M}$ are precisely the $H$-convex sets, for $H=\Omega$, the closure of the set of exposed points of $B^{*}$ (in finite dimensions). Boltyanski and Martini [2] have investigated the question under which conditions the Minkowski sum of $H$-convex sets is again $H$-convex. Their Example 1 seems to contradict our Theorem 3.2. However, H. Martini has informed us that their Examples 1 and 3 are erroneous.

We will now prove a characterization of the finite dimensional polyhedral norms for which $\mathcal{M}$ is ball stable. To this end, we define a critical configuration of $B$ as a pair $\left(N,\left\{f_{1}, \ldots, f_{k}\right\}\right)$ consisting of a normal cone $N$ of $B$ of dimension at least two and a set $\left\{f_{1}, \ldots, f_{k}\right\} \subset \Omega$ of vertices of $B^{*}$ such that

$$
\operatorname{dim}\left(N \cap \operatorname{pos}\left\{f_{1}, \ldots, f_{k}\right\}\right)=1 .
$$

Theorem 3.3. Let $X$ be a finite dimensional polyhedral normed space. Then $\mathcal{M}_{X}$ is ball stable if and only if $B$ does not have a critical configuration.

Proof. Suppose that $\left(N,\left\{f_{1}, \ldots, f_{k}\right\}\right)$ is a critical configuration of $B$. As in the proof of Theorem 3.2, we choose $x_{i} \in \operatorname{relint} F\left(B, f_{i}\right)(i=1, \ldots, k)$ and put

$$
P:=\bigcap_{i=1}^{k}\left(B-x_{i}\right),
$$

then $P \in \mathcal{M}_{X}$ and, by Lemma $2.2, N(P, 0)=\operatorname{pos}\left\{f_{1}, \ldots, f_{k}\right\}$. Let $F$ be the face of $B$ with $N(B, F)=N$ and choose $x \in \operatorname{relint} F$. Again by Lemma 2.2, $N(P+B, x)=$ $N(P, 0) \cap N(B, x)$. Since $x \in \operatorname{relint} F$, it follows that $N(P+B, x)=N(B, F) \cap N(P, 0)=$ $N \cap \operatorname{pos}\left\{f_{1}, \ldots, f_{k}\right\}$. By assumption, this cone has dimension one, hence the unique $g \in S^{*} \cap N(P+B, x)$ determines a facet of $P+B$. If we had $P+B \in \mathcal{M}_{X}$, then Lemma 
2.1 would imply that $g \in \Omega$. Since $g \in N(B, F)$, the face $F$ would be a facet and thus $\operatorname{dim} N(B, F)=1$, a contradiction. Thus, $\mathcal{M}_{X}$ is not ball stable.

Conversely, suppose that $\mathcal{M}_{X}$ is not ball stable. Then there exists a set $P \in \mathcal{M}_{X}$, necessarily a polytope, such that $P+B \notin \mathcal{M}_{X}$. By Lemma 2.1,

$$
P+B \neq \bigcap_{f \in \Omega} H^{-}(P+B, f) .
$$

Therefore, the polytope $P+B$ has a facet $F_{1}$ whose determining functional, say $g \in S^{*}$, is not an element of $\Omega$. Let

$$
F:=F(B, g), \quad G:=F(P, g)
$$

then $F_{1}=F+G$. Choosing a point $x_{1} \in \operatorname{relint} F_{1}$, we can represent it as $x_{1}=x+y$ with $x \in \operatorname{relint} F$ and $y \in \operatorname{relint} G$. Therefore, by Lemma 2.2,

$$
N(P+B, F)=N\left(P+B, x_{1}\right)=N(P, y) \cap N(B, x)=N(P, G) \cap N(B, F) .
$$

By Lemma 2.1, the polytope $P \in \mathcal{M}_{X}$ can be represented as $P=\bigcap_{f \in \Omega} H^{-}(P, f)$. The face $G$ is an intersection of facets of $P$. Hence, there are $f_{1}, \ldots, f_{k} \in \Omega$ such that

$$
N(P, G)=\operatorname{pos}\left\{f_{1}, \ldots, f_{k}\right\}
$$

Since $F_{1}$ is a facet of $P+B$, we have

$$
1=\operatorname{dim} N\left(P+B, F_{1}\right)=\operatorname{dim}\left(N(B, F) \cap \operatorname{pos}\left\{f_{1}, \ldots, f_{k}\right\}\right) .
$$

Since $F=F(B, g)$ and $g \notin \Omega$, the face $F$ is not a facet, hence $\operatorname{dim} N(B, F) \geq 2$. Thus, $\left(N(B, F),\left\{f_{1}, \ldots, f_{k}\right\}\right)$ is a critical configuration.

As mentioned earlier, $\ell_{1}^{d}$ is an example of a space in which $\mathcal{M}$ is ball stable, but not stable.

\section{MAZur SETS In POLYhedral SPACES}

The family $\mathcal{M}$ of all intersections of balls has two nice subfamilies, both in the core of Minkowski's convexity theory: convex bodies of constant width and diametrically maximal sets [5]. In addition to these classical examples, the class $\mathcal{P}$ of Mazur sets (see the definition in the introduction) is another interesting subfamily of $\mathcal{M}$. It is known that sets of constant width are always Mazur sets while this is not the case for diametrically maximal sets [14]. The space is called a Mazur space if $\mathcal{P}=\mathcal{M}$.

It was shown in [9] that the only Mazur sets in $\ell_{1}^{d}$ are one-pointed sets and balls. The main purpose of this section is to give a characterization of the finite dimensional 
polyhedral spaces satisfying this curious property. To this end, the Mazur sets in a finite dimensional polyhedral space are determined in the following theorem.

Let $P, Q \in \mathcal{K}$ be polytopes. We say that the normal fan of $P$ is coarser than the normal fan of $Q$ if the normal fan of $Q$ is a refinement of the normal fan of $P$, that is, every normal cone of $P$ is a union of normal cones of $Q$. A summand of a polytope $P$ is a compact convex set $K$ of positive dimension for which there exists a compact convex set $M$ of positive dimension such that $P=K+M$. Summands of polytopes are necessarily polytopes ([18], p. 143).

Theorem 4.1. For a convex body $K \in \mathcal{K}$ of positive dimension in a polyhedral space $X$, the following conditions are equivalent:

(a) $K$ is a Mazur set.

(b) $K$ is a polytope whose normal fan is coarser than the normal fan of $B$.

(c) $K$ is homothetic to a summand of $B$.

Proof. We begin with an elementary preparation.

Claim. Let $C \subset X$ be a closed convex cone, let $f \in X^{*}$ be a functional such that $C \cap f^{-1}(0)=\{0\}$. Let $K \in \mathcal{K}$ be a convex body such that to every $\varepsilon>0$ there exists a vector $t_{\varepsilon} \in X$ with

$$
K+t_{\varepsilon} \subset C \quad \text { and } \quad\left(K+t_{\varepsilon}\right) \cap f^{-1}(-\varepsilon) \neq \emptyset .
$$

If $x \in K$ is such that $f(x)=\max f(K)$, then $0 \in K-x \subset C$.

For the proof, we observe that $C \subset f^{-1}(-\infty, 0)$. If $t_{\varepsilon}$ satisfies $(3)$, then $x+t_{\varepsilon} \in$ $C \cap f^{-1}(-\varepsilon, 0)$. Choosing a sequence $\left(\varepsilon_{i}\right)_{i \in \mathbb{N}}$ with $\varepsilon_{i} \rightarrow 0$, we see from

$$
\bigcap_{i \in \mathbb{N}}\left[C \cap f^{-1}\left(-\varepsilon_{i}, 0\right)\right]=\{0\}
$$

that $t_{\varepsilon_{i}} \rightarrow-x$ and, hence, $0 \in K-x \subset C$, which proves the claim.

Now let $K \in \mathcal{K}$ be a Mazur set. Let $N(K, x)$ be a normal cone of $K$, and let $f \in N(K, x) \cap S^{*}$; then $x \in F(K, f)$. The support set $F=F(B, f)$ is a face of the polytope $B$. Let $\varepsilon>0$ be given. The hyperplane $f^{-1}(h(K, f)+\varepsilon)$ does not meet $K$. Since $K$ is a Mazur set, there exists a ball $B_{\varepsilon}=\lambda_{\varepsilon} B+t_{\varepsilon}, \lambda_{\varepsilon}>0$, with $K \subset B_{\varepsilon} \subset f^{-1}(-\infty, h(K, f)+\varepsilon)$.

Choose $y \in \operatorname{relint} F$ and let $y_{\varepsilon}:=\lambda_{\varepsilon} y+t_{\varepsilon}$. Then $K-y_{\varepsilon} \subset S\left(B_{\varepsilon}, y_{\varepsilon}\right)=S(B, y)$, the latter by an obvious property of support cones. Moreover, $f(x)=h(K, f) \leq f\left(y_{\varepsilon}\right) \leq$ $h(K, f)+\varepsilon$ and thus $-\varepsilon \leq f\left(x-y_{\varepsilon}\right) \leq 0$. In particular, $K-y_{\varepsilon} \cap f^{-1}(-\varepsilon) \neq \emptyset$.

Since $y \in \operatorname{relint} F$, the support cone $S(B, y)$ is a direct sum, $S(B, y)=X_{1} \oplus C$, where the subspace $X_{1}$ is a translate of the affine hull of $F$, and $C$ is a pointed convex cone in a subspace $X_{2}$ complementary to $X_{1}$. Let $\pi_{2}$ be the canonical projection to $X_{2}$. Writing 
$-y_{\varepsilon}=t_{1, \varepsilon}+t_{2, \varepsilon}$ with $t_{i, \varepsilon} \in X_{i}$, we get from the claim (with $X$ replaced by $X_{2}$ and $K$ replaced by $\left.\pi_{2}(K)\right)$ that $0 \in \pi_{2}(K)-\pi_{2}(x) \subset \pi_{2}(S(B, y))$. Since $K+t \subset S(B, y)$ for all $t \in X_{1}$, this implies $0 \in K-x \subset S(B, y)$. From this, we get $S(K, x)=S(K-x, 0) \subset$ $S(B, y)$. By duality, it follows that $N(B, y) \subset N(K, x)$; moreover, $f \in N(B, y)$. Since $N(K, x)$ was an arbitrary normal cone of $K$, this shows that every normal cone of $K$ is a union of normal cones of $B$. In particular, $K$ has only finitely many normal cones and hence is a polytope. The normal fan of this polytope is coarser than the normal fan of $B$. Thus, (b) holds.

The equivalence of (b) and (c) may be 'folklore', but in default of a suitable reference we give a proof of the necessity. Suppose that (b) holds. Let $f \in S^{*}$ be such that the face $F=F(K, f)$ is an edge of $K$. Since the normal fan of $K$ is coarser than the normal fan of $B$, there exists a normal cone $N$ of $B$ such that $f \in N \subset N(K, F)$. There exists a face $G$ of $B$ with $N=N(B, G)$. Since $N(B, G) \subset N(K, F)$, the face $G$ contains a line segment parallel to the edge $F$. Since there are only finitely many such pairs $(F, G)$, we can choose a number $\lambda>0$ with the following property: whenever $F(\lambda K, f)\left(f \in S^{*}\right)$ is an edge of $\lambda K$, then the support set $F(B, f)$ contains a translate of $F(\lambda K, f)$. Now it follows from [18, Theorem 3.2.8] that $\lambda K$ is a summand of $B$. Thus (c) holds.

Suppose that (c) holds. Then there are a number $\lambda>0$ and a convex body $M$, necessarily a polytope, such that $B=\lambda K+M$. Then, $K+\lambda^{-1} M=\lambda^{-1} B$. Let $f \in X^{*}$ and $\mu \in \mathbb{R}$ be such that $\sup f(K)<\mu$. We choose $y \in \lambda^{-1} M$ satisfying $f(y)=\sup f\left(\lambda^{-1} M\right)$ and consider the ball $C=-y+\lambda^{-1} B$. Since $0 \in \lambda^{-1} M-y$ and

$$
K+\left(\lambda^{-1} M-y\right)=-y+\lambda^{-1} B
$$

we see that $K \subset C$. Finally,

$$
\begin{aligned}
\sup f(C) & =f(-y)+\sup f\left(\lambda^{-1} M\right)+\sup f(K) \\
& =\sup f(K)<\mu
\end{aligned}
$$

which, since $f$ and $\mu$ were arbitrary, proves that $K$ is a Mazur set.

Corollary 4.2. A finite dimensional polyhedral space $X$ is a Mazur space if and only if $\mathcal{M}_{X}$ is stable.

Proof. A useful property of $\mathcal{P}$ is that it is stable under Minkowski sums in any normed space [8]. Hence, in every Mazur space $X$, the set $\mathcal{M}_{X}$ is stable. Conversely, assume that $X$ is a finite dimensional polyhedral space and $\mathcal{M}_{X}$ is stable. Then it follows from Theorem 3.2 that $X$ is of the form $X=X_{1} \oplus_{\infty} \cdots \oplus_{\infty} X_{k}$ with $\operatorname{dim} X_{i} \leq 2$ and each $X_{i}$ polyhedral. If $K \in \mathcal{M}_{X}$, then $K=K_{1} \oplus \cdots \oplus K_{k}$ with $K_{i} \in \mathcal{M}_{X_{i}}$, by Proposition 3.1. Each $X_{i}$ is a Mazur space ([8], Theorem 6.5), hence by Theorem 4.1, $K_{i}$ is homothetic to a summand of $B_{i}$ (the unit ball of $X_{i}$ ). This means that there exist a number $\lambda_{i}>0$ and a convex body $M_{i} \subset X_{i}$ with $B_{i}=\lambda_{i} K_{i}+M_{i}(i=1, \ldots, k)$. Choosing a number $\lambda$ with 
$0<\lambda<0$, we have $B_{i}=\lambda K_{i}+\left(\lambda_{i}-\lambda\right) K_{i}+M_{i}$ and hence

$$
B_{1} \oplus \cdots \oplus B_{k}=\lambda\left(K_{1} \oplus \cdots \oplus K_{k}\right)+\left[\left(\left(\lambda_{1}-\lambda\right) K_{1}+M_{1}\right) \oplus \cdots \oplus\left(\left(\lambda_{k}-\lambda\right) K_{k}+M_{k}\right)\right] .
$$

Thus, $K$ is homothetic to a summand of $B=B_{1} \oplus \cdots \oplus B_{k}$ and is, therefore, a Mazur set. This shows that $X$ is a Mazur space.

Our final result shows that the property that balls and one-pointed sets are the only Mazur sets is not so rare. Here the notion of indecomposable polytopes plays a role. A convex body $K \in \mathcal{K}$ is indecomposable if a representation $K=M+L$ with convex bodies $M, L \in \mathcal{K}$ is only possible if $M$ and $L$ are either one-pointed or homothetic to $K$.

Corollary 4.3. For a polyhedral space $X$, the following conditions are equivalent:

(a) The only Mazur sets in $X$ are balls and one-pointed sets.

(b) The unit ball $B$ is indecomposable.

Proof. Suppose that (b) holds and that $K$ is a Mazur set. By Theorem 4.1, $K$ is homothetic to a summand of $B$. Since $B$ is indecomposable, $K$ can only be a trivial summand, that is, either one-pointed or homothetic to $B$.

Conversely, suppose that (b) does not hold. Then there are two convex bodies $K, M$, neither one-pointed nor homothetic to $B$, such that $B=K+M$. These bodies are polytopes. It follows from Theorem 4.1 that $K$ and $M$ are Mazur sets. Thus (a) does not hold.

If we assume that $d \geq 3$ and endow the set of all isometry classes of $d$-dimensional normed spaces with the Banach-Mazur metric, then the subset of classes of normed spaces with only trivial Mazur sets is dense. This follows from Corollary 4.3, together with the facts that every every simplicial polytope of dimension at least three is indecomposable ([18, Corollary 3.2.13]) and that every convex body can be approximated arbitrarily closely by simplicial polytopes.

\section{REFERENCES}

[1] V. G. Boltyanski, Helly's theorem for H-convex sets, Soviet Math. Doklady 17 (1) (1976), 78-81.

[2] V. G. Boltyanski and H. Martini, Minkowski addition of H-convex sets and related Helly-type theorems, J. Combin. Theory Ser. A, 103 (2003), 323-336.

[3] V. G. Boltyanski, H. Martini and P. S. Soltan, Excursions into Combinatorial Geometry, Springer, Berlin 1997.

[4] A. L. Brown, Suns in polyhedral spaces, Seminar of Mathematical Analysis (Malaga/Seville, 2002/2003), 139-146, Colecc. Abierta, 64, Univ. Sevilla Secr. Publ., Seville, 2003.

[5] G. D. Chakerian and H. Groemer, Convex bodies of constant width, in Convexity and its Applications, P. Gruber and J. Wills, Eds, Birkhäuser, (1983), 49-96.

[6] D. Chen and B.-L. Lin, On B-convex and Mazur sets of Banach spaces, Bull. Polish Acad. Sci. Math. 43 (3) (1995), 191-198. 
[7] R. Giles, D. A. Gregory and B. Sims, Characterization of normed linear spaces with Mazur's intersection properties, Bull. Austral. Math. Soc. 18 (1978), 105-123.

[8] A. S. Granero, J. P. Moreno and R. R. Phelps, Convex sets which are intersections of closed balls, Adv. Math. 183 (2004), 183-208.

[9] A. S. Granero, J. P. Moreno and R. R. Phelps, Mazur sets in normed spaces, Discrete Comput. Geom. 31 (2004), 411-420.

[10] M. Jiménez Sevilla and J. P. Moreno, A note on porosity and the Mazur intersection property, Mathematika 47 (2000), 267-272.

[11] S. Hansen, A generalization of a theorem of Sylvester on the lines determined by a finite point set, Math. Scand. 16 (1965), 175-180.

[12] S. Mazur, Über schwache Konvergenz in den Räumen (L $\left.{ }^{p}\right)$, Studia Math. 4 (1933), 1281-33.

[13] J. P. Moreno, Semicontinuous functions and convex sets in $C(K)$ spaces, J. Austral. Math. Soc. 81 (2006), 1-11.

[14] J. P. Moreno, P. L. Papini and R. R. Phelps, Diametrically maximal and constant width sets in Banach spaces, Canad. J. Math. 58 (4) (2006), 820-842.

[15] J. P. Moreno and R. Schneider, Continuity properties of the ball hull mapping, Nonlinear Anal. 66 (2007), 914-925.

[16] T. Motzkin, The lines and planes connecting the points of a finite point set, Trans. Amer. Math. Soc. 70 (1951), 415-464.

[17] R. R. Phelps, A representation theorem for bounded convex sets, Proc. Amer. Math. Soc. 11 (1960), 976-983.

[18] R. Schneider, Convex Bodies: the Brunn-Minkowski Theory, Encyclopedia of Mathematics and its Applications, 44, Cambridge University Press, Cambridge 1993.

[19] W. Weil, Über die Projektionenkörper konvexer Polytope, Arch. Math. 22 (1971), 664-672.

JOSÉ PEDRO MORENO: Dpto. Matemáticas, Facultad de Ciencias, Universidad Autónoma DE MADRID, MADRID 28049, SPAIN. josepedro.moreno@uam.es

ROLF SCHNEIDER: Mathematisches Institut, Albert-Ludwigs-Universität, D-79104 FREIBURG I. BR., GERMANY. rolf.schneider@math.uni-freiburg.de 